\title{
Dual-Channel Haptic Synthesis of Viscoelastic Tissue Properties Using Programmable Eddy Current Brakes
}

\author{
Andrew H. C. Gosline and Vincent Hayward \\ Haptics Laboratory, Centre of Intelligent Machines, McGill University, Canada
}

\section{Abstract}

We describe a novel method for haptic synthesis of viscoelastic responses which employs a dual-channel haptic interface. It has motors that generate torque independently from velocity and brakes that generate viscous torque independently from position. This way, twice as many states are directly accessible, which reduces reliance on observation and feedback. Torque-generating actuators, e.g. DC motors, are well known. For the viscous actuators, we use eddy current brakes as programmable, linear, non-contact, physical dampers. By decomposing a mechanical impedance to be realized into viscous and elastic components, we can dedicate each actuator to that component it is ideally suited to synthesize, dampers for the viscous component, and motors for the elastic component. The decomposition is in general not unique so it possible to select the option that takes the best advantage of the hardware. Experimental results show that this technique can render a variety of viscoelastic models without the artifacts that can occur when synthesizing viscous components on conventional haptic interfaces. The synthesized mechanical impedances have guaranteed passivity, and can have arbitrarily high or low viscous and elastic components.

\section{Introduction}

Medical virtual environments, analogues of flight simulators for pilots, provide an accurately monitored and safe method for medical training. Students and practitioners alike can learn new skills and rehearse difficult and complicated surgical procedures. Haptic rendering (also referred to as force feedback) is a vital component of these interactive simulations because in a number of cases, much of the information necessary to perform surgical procedures is extracted through touch. Examples include simulators for procedures such as laparoscopy (Basdogan et al., 2001) and brachytheraphy (Goksel et al., 2006). Other contemporary and emerging applications are described in recent surveys (Harders, 2008; Basdogan et al., 2007).

Most tissues exhibit viscoelastic behavior, which complicates the simulations above purely elastic deformations because the rate of deformation must enter in the formulation. Creep, relaxation and hysteresis are examples of deformation-rate-dependent phenomena that are germane to viscoelastic tissues (Fung, 1993). While simulating rate-dependent phenomena can have a great impact on the visual realism of a simulation, the same is true for haptic realism. Inserting and retracting a needle in an elastic medium does not feel the same as doing the same in a viscoelastic medium. The role of realism in simulators may be discussed, nevertheless, it is beyond debate that surgical simulators that are capable of synthesizing a wide range of tissue behaviors offer far more pedagogical options than those that don't. Haptic rendering is possible only if complex mechanical impedances can be precisely synthesized.

A variety of methods have been proposed to display viscoelasticity for surgery simulators, most of which use networks of discrete springs and dampers 
to model viscoelastic effects, or the Finite Element Method (FEM), as used by Astley and Hayward (1998), Sedef et al. (2006), or Brouwer et al. (2007). Alternatively, Discrete Green's Functions have been used by Schoner et al. (2004). Recently, an explicit simulation and interpolation approach using radial basis functions were described by Höver et al. (2009). Regardless of what numerical method authors have used to compute a viscoelastic response, the result yields a force relationship that is dependent on both the velocity and the position of the virtual instrument. Velocity measurement or estimation is therefore an integral part of the problem of realizing a haptic simulation of viscoelastic mechanical behavior.

\section{Options For The Measurement And Estimation Of Velocity}

With impedance-type devices - the causality of choice for most surgical simulators which, when needed, must be able to present very low impedances to the user - closing the loop through a velocity measurement or a velocity estimate is subject to tradeoffs that are similar to those needed when closing the loop through a position sensor: one must cope with noise, sampling, delay, mechanical modes, as well as time-varying and configuration-dependent system dynamics.

To give a sense of the requirements, it is useful to consider that inserting a needle is an act that normally occurs at low velocity, viz. $10^{-2} \mathrm{~m} \cdot \mathrm{s}^{-1}$. Taking the Phantom 1.0 as an example of a hardware platform (the highest performing model in the range), this device has an advertised resolution of $30 \times 10^{-6} \mathrm{~m}$. This number is arrived at by dividing the encoder resolution by the mechanical transmission ratio, which indicates that its practical resolution is necessarily lower. For the sake of example, we can retain this theoretical resolution, given that it is a resolution guaranteed to be never achieved in pratice. If we chose to update forces at a rate of $1 \mathrm{kHz}$, the basic quantum of velocity achieved by a single backwards difference estimation is $3 \times 10^{-2} \mathrm{~m} \cdot \mathrm{s}^{-1}$, or approximately three times larger than the velocity we wish to measure.

\subsection{Inverse-time methods}

One option is to use the so-called inverse-time velocity estimation method where a velocity estimate is obtained by measuring the time elapsed between two encoder pulses rather than the number of pulses between two clock interrupts (Wallingford and Wilson, 1977; Cavusoglu et al., 2002). For medical simulations, however, this method suffers from critical shortcomings. Estimates are obtained at a rate dictated by the pulses of the encoder and not by the system clock. Causal interpolation schemes to reconcile sampling rates mismatch will inevitably introduce a variable delay, destabilizing in closed-loop. Delay-compensating, non-causal predictive interpolation schemes could be contemplated, but even then, estimates are necessarily erroneous around each velocity reversal and have the wrong sign until a new pulse is sensed.

\section{$2.2 \quad$ Velocity smoothing}

When sampling an encoder counter on a regular clock signal, the standard approach is to low-pass the velocity estimates to filter out the noise. The tradeoffs involving types of filters and filter orders are much too numerous to be discussed here, but in general phase delay and noise attenuation always create opposing requirements. The implementer can also apply various types of tracking estimators to obtain a better velocity signal but invariably, tradeoffs must be made ( $\mathrm{Su}$ et al., 2006), and/or a system model must be assumed (Bélanger et al., 98). Tradeofffree adaptive FIR filters can be helpful (Janabi-Sharifi et al., 2000). In our experience those filters permit the level of simulated viscosity to be raised by an order of magnitude, roughly, but there is always a point where limit cycles occur and their onset is difficult to predict.

\section{$2.3 \quad$ Velocity sensing}

Current haptic interface hardware typically does not provide velocity sensing, but one could consider designing or retrofitting devices with velocity sensors. The mechatronic method of choice for sensing velocity is the tachometer. It could be an option to provide it together with the indispensable position sensor. However, by principle, they do not operate well at low velocities. A high-quality tachometer (Model 
DCT 22, Maxon Motors ag, Sachseln, Switzerland) mounted on the motor shaft of a Phantom 1.0 would give less than $1 \mathrm{mV}$ (with $6 \%$ of ripple) for the example given above, which poses an instrumentation challenge without eliminating filter design tradeoffs.

\section{Options for Transducing Viscos- ity Directly}

All these options, velocity measurement, model-free estimation, or state reconstruction yield artifacts such as delayed response or oscillations due to the approximations that each method must contend with. It is therefore natural to examine the possibility of producing viscosity directly in a programmable fashion, thereby bypassing the necessity to estimate or measure velocity.

\subsection{Fluid viscosity}

The most common industrial approach is to take advantage of the viscosity of fluids. Such systems involve a hydrostatic pump or a piston pushing fluid through an electromagnetic (or electrorheological fluidic) valve. There is a number of reasons why this approach is not directly suitable to create programmable viscosity for medical simulations. Chiefly among them is the necessity to establish turbulent flow in the valve orifice, giving a quadratic relationship between velocity and force that has zero slope at the origin. The resulting strongly nonlinear damping relationship would not fit conveniently into robotic control frameworks (Blackburn et al., 1960).

\subsection{Electromagnetic motors}

Another approach is to leverage electromotive forces. These forces are present in any electrical machine. Conductors moving in magnetic fields always induce an electromotive force. If a path is provided for current to flow, the current interacts with the magnetic field to oppose motion. Nominally, because of the linear nature of Lorentz' law, the torque is proportional to the angular velocity, creating a viscouslike torque that opposes motion. DC motors which are frequently used to make haptic devices, whether of the wound or coreless types, exhibit this effect. For example, shorting the terminals of the motors of a Phantom device (model RE25, Maxon Motors ag, Sachseln, Switzerland) creates a viscous coefficient at the tip which evaluates to approximatively $0.6 \mathrm{~N} \cdot \mathrm{s} \cdot \mathrm{m}^{-1}$. At $10^{-2} \mathrm{~m} \cdot \mathrm{s}^{-1}$, the viscous force is only $6 \mathrm{mN}$ which too small for a realistic simulation.

Of course, it is possible to consider the design of a feedback circuit to modulate the effective internal resistance of the motor, so that the induced current is higher than that permitted by the natural resistance of the windings, but such a design is delicate to achieve. If the motor is brushed, its terminal resistance varies considerably as the motor turns (1:10 ratios are not uncommon) and when the brushes slip from segment to segment on the commutator. Moreover, each time a new winding is connected and another is disconnected, the circuit would have to deal with a new inductor circuit. Another problem is the drift of resistance when the windings heat and cool.

Commutation-free electromagnetic actuators can be considered to alleviate these problems, i.e. limited angle torquers or galvanometric motors. Nevertheless, the feedback design would have to be able to modify the electrical behavior of a motor such that, once converted to the mechanical domain, the viscous component and the elastic components of the resulting mechanical impedance can be independently specified. Undoubtably, such a feedback controller would have to be realized with mixed signal technology, partly digital, partly analog, to be truly programmable. Recently, a circuit able to add modest programmable electrical damping over a limited range of motion with a brushless motor was presented (Weir et al., 2008). If such circuits can be realized to achieve wide-range modulation of damping independently from torque, then the methods described in Section 5 would apply directly to this approach.

\subsection{Taking the bull by the horns}

It is possible to take advantage of Lorentz' law to generate programmable viscosity directly. Devices that are quite appropriate to realize this function are eddy current brakes or ECB's. With this approach, velocity dependent phenomena can be synthesized, keeping the velocity signal out of the feedback loop (Gosline et al., 2006).

ECB's are simple magnetic devices consisting of a conductor that moves through a magnetic field. Eddy currents are induced as a result of the motion in the conductor itself, and the interaction between 
these currents and the applied magnetic field generates a resistive force that is proportional to the relative velocity, according to the Lorentz' Force Law. Although the phenomena are difficult to analyze for complex geometries (Wiederick et al., 1987), the underlying relationship between velocity and force can be illustrated with several simplifying assumptions. Following Heald, 1988, the induced current density in the conductor, $J\left[\mathrm{~A} \cdot \mathrm{m}^{-2}\right]$, is a function of the velocity, $v\left[\mathrm{~m} \cdot \mathrm{s}^{-1}\right]$, the specific resistivity of the material, $\rho[\Omega \cdot \mathrm{m}]$, the electrostatic field of Coulomb charge induced within the conductor, $E[\mathrm{~V}]$, and the magnetic field, $B[\mathrm{~T}]$, that is

$$
J=\frac{1}{\rho}(E+v \times B) .
$$

Computing the current paths given the motion and applied field is complex, and is beyond the scope of this paper. Closed form solutions exist for simple geometries, such as an infinite conductor. Regardless of the eddy current flow regime that ensues, the braking force, $F[\mathrm{~N}]$ can be computed by integrating over the pole projection volume, $\tau\left[\mathrm{m}^{3}\right]$

$$
F=\int J \times B d \tau
$$

Assuming a rectangular pole with width $w[\mathrm{~m}]$ and length $l[\mathrm{~m}]$, neglecting the air gap, and assuming a constant thickness of the conductor, $d[\mathrm{~m}]$, the eddy current brake force is then

$$
F=-\alpha \frac{d l w}{\rho} B^{2} v,
$$

where $\alpha$ is a correction factor related to the eddy current distribution through the pole projection volume. For further details, refer to Heald, 1988. According to (3), the drag force varies linearly with velocity, and prior work has found this to be true for low speeds (Heald, 1988; Anwar, 2002; Lee and Park, 2002). As the relative speed increases, however, the induced magnetic field from the eddy currents becomes too large to neglect, and the braking force begins to diminish. Because haptic interfaces are designed for interaction with humans, the velocity that an interface is subjected to is typically low, of order $200 \mathrm{~mm} \cdot \mathrm{s}^{-1}$ (Lederman et al., 1999), and prior work has shown that eddy current brakes do behave linearly at speeds typical of haptic interaction (Gosline et al., 2006).
Fig. 1 shows an experimental eddy current brake used to perform experimental validation of the methods described in this article. The figure shows a toroidal electromagnet capable of producing a field of about $1.0 \mathrm{~T}$ inside a $3.18 \mathrm{~mm}$ air-gap. A torque motor drives a rotating arm directly so that a user can interact with a manipuladum moving on the arc of a circle. The rotating arm supports a $1.59 \mathrm{~mm}$ thick, $50 \mathrm{~mm}$ radius aluminum annulus that moves concentrically with the motor. When the electromagnet is activated, eddy currents - also called Foucault currents - induced in the annulus create a viscous torque that adds to the torque created by the torque motor. The preferred geometry of such viscous brakes is more fully described by Gosline and Hayward (2008) .

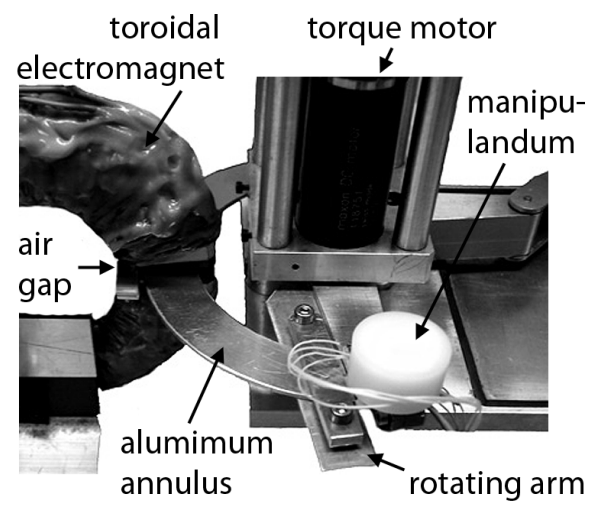

Figure 1: Experimental eddy current brake. This system comprises a blade in the shape of an annulus section of average radius $50 \mathrm{~mm}$ moving in the air gap of a toroidal electromagnet. The brake is coupled directly to a coreless DC motor.

ECB's synthesize viscosity without any mechanical contact nor any electrical contact. By their physics, for all practical purposes, they create perfectly accurate viscosity. This has two consequences. When synthesizing the viscoelastic response of a virtual environment, the more they contribute, the more accurate is the response, without any tradeoff. Secondly, since the passivity of a system can only increase with the addition of any combination of dissipative elements, there is no tradeoff either in attempting to maximize their use. The optimal use of their properties is explored in the next sections. 


\section{Modeling Viscoelasticity}

Classical models of viscoelasticity usually consist of a network of discrete springs, (Fig. 2a) and dampers (Fig. 2b) to reproduce the relaxation, creep and hysteresis characteristics of common materials. Figs. 2c and $2 \mathrm{~d}$ show the two simplest viscoelastic models, the Kelvin-Voight model and Maxwell model respectively. While the Kelvin and Maxwell models exhibit rate dependence, creep, and relaxation, authors generally prefer more complex models for fitting real data. Fig. 2e shows a $2^{\text {nd }}$ order Maxwell model that is a good example of a more complex viscoelastic constitutive law. This model was shown to provide a good fit for biological tissue behavior, and is described as a "general viscoelastic solid" (Christensen, 1971). Recently, Brouwer et al. (2007) used a $2^{\text {nd }}$ order model to fit experimental data from a porcine brain deformation. With a similar model, Wang and Hayward (2007) obtained a good fit for the in-vivo behavior of fingertip skin.

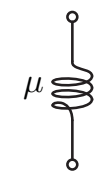

a

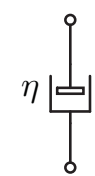

b

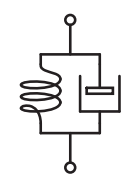

C

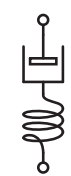

d

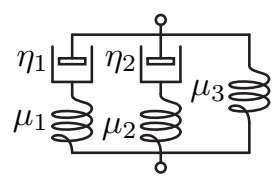

e
Figure 2: Single Spring (a). Damper (b). Kelvin (c). Maxwell (d). $2^{\text {nd }}$ order model (e).

Our immediate aim is to investigate the simulation and the solution of spring-damper networks so that they may be physical re-synthesized with a haptic display for a user to experience them as if she/he was interacting with real tissues.

Because of its practical importance we focus on the $2^{\text {nd }}$ order generalized Maxwell model. If the springs and dampers can be assumed to be linear, the Laplace transform method can be used to solve for the deformation response or for the force response (Findley et al., 1976). In the case of the $2^{\text {nd }}$ order model, the total stress in the element, $\sigma[\mathrm{Pa}]$, is the summation of the stresses in each of the load paths,

$$
\sigma=\sigma_{1}+\sigma_{2}+\sigma_{3} .
$$

For each element, the stress is related to strain, $\epsilon[\%]$, by,

$$
\dot{\epsilon}_{1}=\frac{\dot{\sigma}_{1}}{\mu_{1}}+\frac{\sigma_{1}}{\eta_{1}}, \quad \dot{\epsilon}_{2}=\frac{\dot{\sigma}_{2}}{\mu_{2}}+\frac{\sigma_{2}}{\eta_{2}}, \quad \sigma_{3}=\mu_{3} \epsilon_{3} .
$$

With the assumption of time independence, each differential equation can be solved independently. It follows that, given identical initial conditions, each solution can be combined in the time domain, such that the stress to a ramp input of strain (constant strain rate) at $t=0$ is:

$\sigma=\dot{\epsilon} \eta_{1}-\dot{\epsilon} \eta_{1} e^{-\left(\mu_{1} / \eta_{1}\right) t}+\dot{\epsilon} \eta_{2}-\dot{\epsilon} \eta_{2} e^{-\left(\mu_{2} / \eta_{2}\right) t}+\mu_{3} \dot{\epsilon} t$.

Note that (6) contains two exponential decay functions that correspond to the separate Maxwell elements, hence the nomenclature that it is a $2^{\text {nd }}$ order model. The time-domain solution to a given viscoelastic constitutive law is useful to verify the accuracy of a simulation update law using the same model. In Section 5, we use these results to verify that that the discrete realization of viscoelastic functions agree with the closed form solutions.

\section{Realization of Viscoelastic Functions for Haptic Synthesis}

In a haptic synthesis framework, a force update law is required given inputs of position and velocity,

$$
f_{k}=f\left(u_{k, k-1, \ldots}, \dot{u}_{k, k-1, \ldots}\right),
$$

where $f_{k}$ is the force to be returned by the device at step $k, u_{k}$ is the $k^{\text {th }}$ position measurement from the device, and $\dot{u}_{k}$ is the $k^{\text {th }}$ velocity estimation or measurement. Input and output could be assumed to occur in synchrony if the sampling period is sufficiently long compared to the conversion delays. However, in most practical realizations, it is often preferred to increase the sampling as high as it is feasible. The update law is then

$$
f_{k}=f\left(u_{k-1, k-2, \ldots}, \dot{u}_{k-1, k-2, \ldots}\right) .
$$

We now examine the realization of viscoelastic constitutive laws into an update law in the form of (8). Earlier, we discussed different approaches for realizing devices that would be more capable than systems based on measuring position only and producing force only.

With the haptic simulation of viscoelastic behaviors in mind, we examined two possibilities. The first was to improve the measurement or estimation of velocity at low speeds. The second was to consider programmable viscous actuators in addition to standard motors (or perhaps the electronic modification 
of the electromechanical behavior of standard motors). We concluded that the later approach would be more straightforward to realize. This is the assumption we adopt for the remainder of this paper. It should be noted however that similar manipulations could be carried out to account for the availability of high quality velocity signals, that is down to $10^{-3}$ or $10^{-4} \mathrm{~m} \cdot \mathrm{s}^{-1}$.

In the next subsections we examine basic cases and look at the realization of a $2^{\text {nd }}$ order model that can take best advantage of the physical properties of viscous brakes in parallel with torque generating motors. It is also worthwhile noting that the series connection of a torque-generating motor with a viscous brake, in essence a viscous clutch, could also have certain applications but wouldn't be directly applicable to simulating tissue behavior.

At this point, since the interaction with a tissue can be assumed to be effected through a tool, we no longer need to use the notion of stress-strain in the tissue or at its boundary. We can lump these distributed quantities into a force applied to a tool in response to a displacement.

\subsection{Realization of a Kelvin Element}

The realization of a Kelvin element is straightforward due its parallel nature. The interaction with a "virtual wall" in the haptics literature is most often modeled as a contact with a Kelvin element, such that

$$
f_{k}=K_{k} u_{k-1}+B_{k} \dot{u}_{k-1},
$$

where $u_{k}[\mathrm{~m}]$ is the deflection of the spring and $K_{k}\left[\mathrm{~N} \cdot \mathrm{m}^{-1}\right]$ and $B_{k}\left[\mathrm{~N} \cdot \mathrm{s} \cdot \mathrm{m}^{-1}\right]$ are the desired values of stiffness and damping at a particular sampling period. The quantities $K_{k}$ and $B_{k}$ can be allowed to vary provided that they do so slowly compared to $u$ and $\dot{u}$, lest they also participate in the variations of $f$. For the purpose of this paper focused on viscoelastic simulations of tissues, this assumption can be assumed to hold.

A single 1-DOF kelvin element, as described by (9) maps directly to a programmable parallel-connected damper-motor pair. Instead of providing damping by velocity feedback (virtual damping), as would be required for interfaces without programmable physical dampers, two command signals can be sent in parallel. One to the amplifier-motor channel and the other to the amplifier-damper channel. Provided that the brake responds sufficiently fast, the system (and the haptic experience) will remain passive for any value of $K$ and $B$ provided that a minimal damping is commanded (Colgate and Schenkel, 1994). Estimates of this minimum damping can easily be computed in open loop from each sample $K_{k}$ and from the sampling period (Hayward, 2007), or determined incrementally from cycle to cycle (Gosline and Hayward, 2008). A single Kelvin element is the optimal viscoelastic function for our dual-channel haptic interface, as it is a mirror image of the hardware.

Following Findley et al. (1976), the Kelvin model, like the Maxwell model, can be generalized to represent a rich set of behaviors by connecting additional elements, provided that they have different relaxation times. Just like the $2^{\text {nd }}$ order model mentioned in Section 4 is an example of a generalized Maxwell model with two time constants, the $2^{\text {nd }}$ order generalized Kelvin model has two elements in series to represent two retardation times. It is shown in Fig. 3.

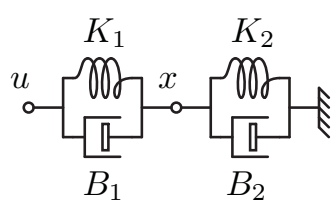

Figure 3: Generalized Kelvin model.

Because the Kelvin elements are in series, they share the same force. For simulation it is necessary to compute the state, $x[\mathrm{~m}]$, that represents the displacement of the elements relatively to the total displacement. The force balance is

$$
K_{1}(u-x)+B_{1}(\dot{u}-\dot{x})=K_{2} x+B_{2} \dot{x} .
$$

Time discretization of (10) leads to an update law for $x$,

$$
\begin{aligned}
x_{k}= & \left(K_{1 k} u_{k-1}+B_{1 k} \dot{u}_{k-1}+\frac{B_{1 k}+B_{2 k}}{\Delta t} x_{k-1}\right) \\
& \left(K_{1 k}+K_{2 k}+\frac{B_{1 k}+B_{2 k}}{\Delta t}\right)^{-1} .
\end{aligned}
$$

The force output can be computed using the left hand side of (10), such that the static spring component $K_{1}(u-x)$ plus a term due to the slow migration of the hidden state $-B_{1} \dot{x}$ can be output by the motors, while the dampers can handle the output of $B_{1} \dot{u}$ without measuring velocity. 
As an additional benefit, it is possible to choose which Kelvin element is used as an observer for the force output. Numerical tests have shown that it is best to select the Kelvin element that is responsible for the largest amount of dissipation within the assembly to maximize the passivity advantage of dual channel rendering. This way, a user forcefully manipulating the simulation will constantly be resisted by the damper - which comes "free" - and not by the motor. This realization generalizes to any number of Kelvin elements in series.

\subsection{Realization of a Maxwell Element}

The realization of a Maxwell element is less straightfoward than that of a Kelvin element because of the additional state which must be computed. To further complicate the matter, there are two possible configurations which are mechanically equivalent but computationally distinct, yielding four different methods to compute the force output of a Maxwell element. Referring to Fig. 4a or 4b, the force output can either be computed by the difference of velocity across the damper, or difference in position across the spring.

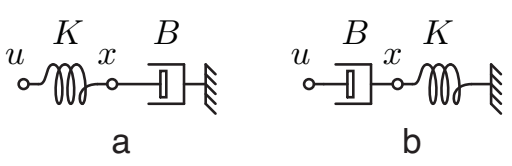

Figure 4: Generalized Maxwell model. The two possible realizations.

In the realization of Fig. 4a, the force balance is:

$$
K(u-x)=B \dot{x} .
$$

Time discretization of (12) leads to an update law for $x$ of the form

$$
x_{k}=\left(K u_{k-1}+\frac{B}{\Delta t} x_{k-1}\right)\left(K+\frac{B}{\Delta t}\right)^{-1} .
$$

Once the state $x$ is known, the output force can either be computed by

$$
f_{k}=B \dot{x}_{k-1}
$$

or by

$$
f_{k}=K\left(u_{k-1}-x_{k-1}\right) .
$$

It is clear from (14) and (15) that this method does not allow to take direct advantage of the motordamper tandem. In the case of (15), there are no velocity dependent terms. Equation (14) is velocity dependent, and would work for an initial contact with a Maxwell element. However, when the velocity decreases, goes to zero, or changes sign, dampers are unable to generate the required transient forces. An alternate method for realizing a Maxwell element is as in Fig. 4b. The force balance is

$$
K x=B(\dot{u}-\dot{x}) .
$$

In this realization, the contribution of $B \dot{u}$ can be directly provided by the physical dampers, leaving the motors to responsible for small and transient elastic correction forces according to $-B \dot{x}$. In this formulation, the update of $x$ is computed by

$$
x_{k}=\left(B_{k} \dot{u}_{k-1}+\frac{B_{k}}{\Delta t} x_{k-1}\right)\left(K_{k}+\frac{B_{k}}{\Delta t}\right)^{-1} \text {. }
$$

Once the state $x$ is known, for the configuration as in Fig. 4b, the force output can either be computed by

$$
f_{k}=K_{k} x_{k}
$$

or by

$$
f_{k}=B_{k}\left(\dot{u}_{k-1}-\dot{x}_{k}\right)
$$

The only hardware-realizable force observation law is (19), since the physical dampers can be used to directly produce the term $B_{k} \dot{u}_{k}$ by physics, and the motor be left to handle the term $-B_{k} \dot{x}_{k}$. This is required for transient elastic components only.

Velocity estimation is still required to compute the state $x$ in (17), risking noisy velocity estimation to make its way into the force output law. However, (17) is an integration process which is robust to noisy inputs. Moreover, in the force observation expression (19), the correction term $-B \dot{x}$ appears only during transients, and acts when the physical dampers also contribute the $B \dot{u}$ term completely in a physically exact manner. As a result, the physical dampers contribute the greatest part of the output force, and the effect of a noisy and/or delayed velocity estimation is minimized.

It is also worthwhile noting that this formulation corresponds exactly to an early suggestion by Colgate and Schenkel (1994) to maintain passivity of a haptic interface by adding excessive physical damping and removing it with negative computational damping. Such approach is guaranteed to err on the side to too much passivity rather than on the side of too little of it. 
Generalized Maxwell models have a plurality of Maxwell elements in parallel. For this arrangement, see Fig. 2e, an intermediate state, $x_{\{1,2, \cdots n\}}$, must be computed for each of the $n$ Maxwell elements connected in parallel. Due to linearity, the contribution from each discrete damper will sum, thus the total damping coefficient is the summation of each discrete damper, and the total motor contribution is the sum of each motor contributions.

\subsection{Closed Form Solution Vs. Realiza- tion of a $2^{\text {nd }}$ Order Maxwell Model}

Fig. 5 shows a comparison between the closed-form solution of a $2^{\text {nd }}$ order Maxwell model (Section 4, Fig. 2e) and the time-discretized realization as described above for contact with a constant strain rate. The upper left panel illustrates that the simulation agrees well with the closed form solution, even using a fixed time step of $200 \mathrm{~ms}\left(K_{1}=12 \mathrm{~N} \cdot \mathrm{m}^{-1} ; K_{2}=\right.$ $10 \mathrm{~N} \cdot \mathrm{m}^{-1} ; K_{3}=5 \mathrm{~N} \cdot \mathrm{m}^{-1} ; B_{1}=5 \mathrm{~N} \cdot \mathrm{s} \cdot \mathrm{m}^{-1} ; B_{2}=$ $6 \mathrm{~N} \cdot \mathrm{s} \cdot \mathrm{m}^{-1}$ ). The same simulation with a time step of $1 \mathrm{~ms}$ would be graphically undistinguishable from the closed-form solution. The lower left panel shows the strain applied, a constant ramp of strain for 4 seconds, followed by a constant strain after 4 seconds. The upper and lower right panels show the contributions of the damper and the motor respectively, see (19). This figure shows the transients necessary to compensate for the physical damping whenever the manipulandum changes velocity. The sharp transients in the motor's contribution are needed due to the sharp changes in velocity that are possible in simulation. In practice, humans accelerate at a finite rate, so under normal operating conditions, the motor's contribution always remains well conditioned.

\subsection{Simulation of a Maxwell Element for Passivity Analysis}

Using the dual-channel realization methods for a single Kelvin element can completely remove the velocity estimation signal from the feedback loop. However, for a Maxwell element, simulation of the intermediate state $x$ using (17) requires that the velocity of the interaction point, $u$, be known. Fig. 6 shows results from a numerical comparison between the closed form solution (grey line), a simulation of conventional rendering using a motor for elastic and viscous components according to (19) (thick black
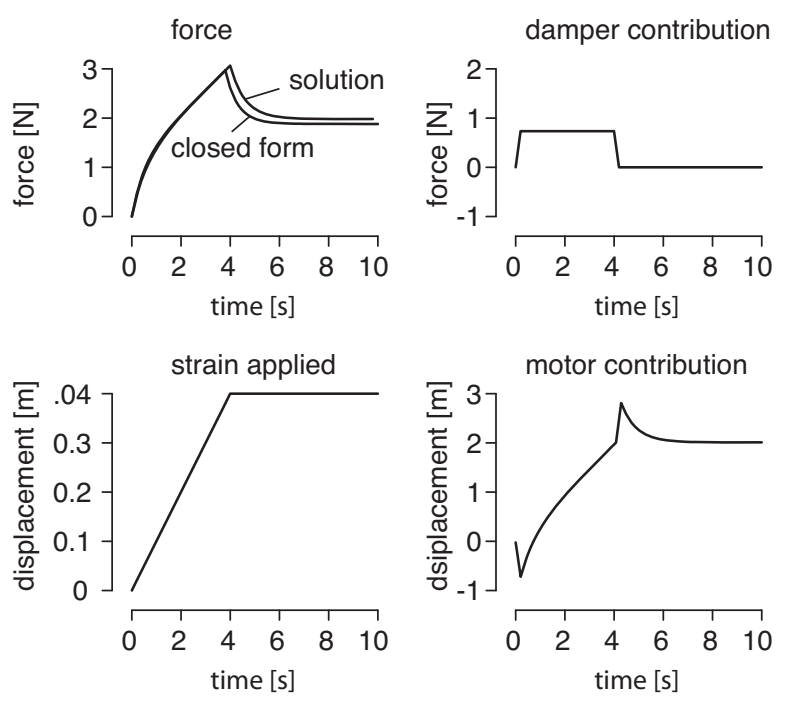

Figure 5: Realized simulation versus closed-form Solution for 2 nd order model. This example was computed with a time step of $200 \mathrm{~ms}$ to exaggerate the error, which still remains small.

line), and a simulation of dual-channel rendering using a motor for elastic and a physical damper for viscous components according to (19) (thin black line). The difference between the conventional and dualchannel cases is that the viscous component, $B \dot{u}$, is computed with a delayed velocity in the conventional case, while with the delay-free velocity in the dualchannel case. In both simulated cases, conventional and dual-channel, $B \dot{x}$ is computed with a delayed velocity by (17). In the simulation, $B=5, K=50$, and $\Delta t=0.001 \mathrm{~s}$. The input, $u$, is modeled as $0.5 \mathrm{~Hz}$ sine wave of velocity, and the velocity estimation is modeled as a $5 \mathrm{~ms}$ delay in velocity.

Note that in the output force plot, the single-motor trace (thick black line) exhibits considerable delay, but the dual-channel output (thin black line) exhibits a reduced force, and a smaller delay due to the correction term $-B \dot{x}$ and the delay-free physical dampers. The energy trace in Fig. 6 is computed using a passivity observer (PO), as described by Hannaford and Ryu (2002):

$$
E_{\text {obsv }}(k)=f(k) v(k) \Delta t .
$$

It is clear from the plots that using a single motor to output viscous components causes the energy trace to return too much energy due to delay in the velocity estimate as well as in the elastic term. By the convention used by Hannadord and Ryu (2002), energy 

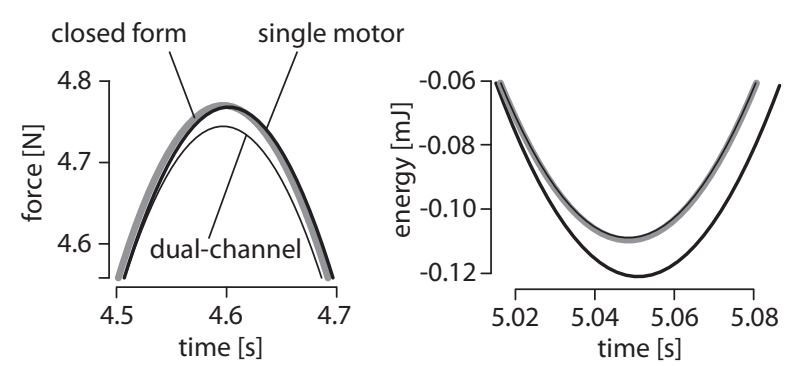

Figure 6: Output force and discrete energy of a single Maxwell element using various velocity estimation models. Thick grey trace is closed form, thin black trace is dual-channel, thick black trace is motor only. It is clear that the single-motor synthesis yields nonpassive energetic behavior whereas the dual channel solution tracks the closed form solution nearly perfectly.

dissipation is positive, so energy traces in the negative regions indicates active behavior. In the energy plot of Fig 6 the energy trace goes lower into the negative energy region and stays there longer than in the other cases. The dual energy behavior of the dualchannel technique indicates nearly perfect behavior but, as predicted, errs toward a slightly over-damped behavior. This finding agrees with prior work, notably that of Colgate and Schenkel (1994). Compensation for physical dissipation removes too much energy, and is thereby guaranteed to be passive.

\section{Experimental Validation}

As this work is primarily aimed at surgery simulation, multidimensional simulation problems should eventually be studied. But for the purpose of this article, we restrict validation to the one-dimensional case since its success is a prerequisite to the feasibility of multidimensional cases. Moreover, it can be observed that numerous medically relevant tooltissue interaction cases occur along one single dimension. Such is the case of needle insertion and catheterization, both of which have dedicated commercially available one-DOF simulators. ${ }^{1}$

As indicated earlier, when employing ECB's for haptic synthesis, fidelity and passivity can only increase in proportion to their overall contribution to the final mechanical impedance experienced by the user, without tradeoff. This is due to the reduction

\footnotetext{
${ }^{1}$ see www.immersion.com/medical
}

of reliance on velocity estimation suffering from delay and quantization effects.

\subsection{Hardware}

The prototype dual-channel haptic interface hardware comprises the Pantograph haptic interface which is retrofitted with concentric annular aluminum sections on each base arm. As shown in Fig. 1, only one axis is used. The $1.59 \mathrm{~mm}$ thick, $50 \mathrm{~mm}$ radius "damper blade" moves through the air gap of a powerful electromagnet driven by a switching amplifiers set in current mode (Model AMC 20A20 Advanced Motion Controls, Camarillo, CA, USA) with a 150 VDC power rail. The motor is a coreless DC motor (Model RE25, 118751, Maxon Motors ag) driven by a linear current amplifier (model LCAM, Quanser Inc., Markham, ON, Canada) with a 27 vDC power rail. The position sensor is a $65 \mathrm{~K}$ counts-perrevolution quadrature optical encoder (model R119, Gurley Precision Instruments, Troy, NY, USA). Signal sampling and reconstruction is done with a ISA bus IO card (model STGII-8, Servo To Go Inc., Indianapolis, IN, USA) on a $2.8 \mathrm{GHz}$ PC running Linux Kernel 2.6 and the Xenomai Realtime Framework.

\subsection{Velocity Estimation}

It is important to gain an appreciation for the magnitude of the noise and delay that is commonplace in haptic simulations with optical encoders. Despite the reliable, drift -free position sensing ability of encoders, raw velocity estimation by backward difference is very noisy, and requires considerable filtering before it can be used in a haptic simulation. As mentioned in the introduction, filtering techniques are required to allow a velocity estimation to be fed back into the synthesis algorithm.

Fig. 7a shows the level of noise that is present with a single backward difference velocity estimation using our high precision encoders at low speed. Each step corresponds to a velocity quantum of 0.95 radians-per-second with many switches. Fig. 7b shows three signals. The first is the quantized position signal. The second is the output of non-causal delay-free 100 samples averaging filter. The third is a filtered signal using a $120 \mathrm{~Hz}$ fourth-order Butterworth FIR low-pass filter. This figure exemplifies the basic tradeoff that every velocity dependent haptic simulation system faces. A smooth signal can be ob- 
tained only at the cost of delay, here, the filtered signal estimates cross zero with approximately $5 \mathrm{~ms}$ of delay. The $120 \mathrm{~Hz}$ filter is necessary to generate virtual damping of the same magnitude than that can be be produced by the ECBs prototypes, approximately $7 \mathrm{mN} \cdot \mathrm{m} \cdot \mathrm{s}$.

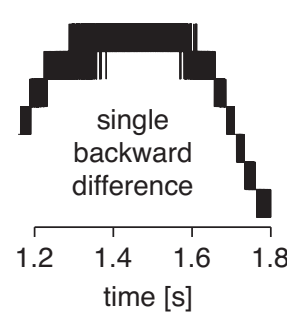

a

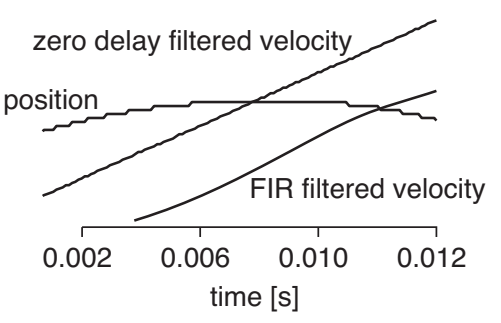

b
Figure 7: Noise and/or delay are associated with approximating velocity from a discretized position signal. Plots are drawn with arbitrary units.

\subsection{Examples of Viscoelastic Behavior Haptic Synthesis}

Experiments were performed to demonstrate the use of physical damping for three different examples of viscolastic behavior. The first case is the synthesis of a Kelvin model that exemplifies the difficulties associated with the use of computational viscosity and the second demonstrates the synthesis of a Maxwell model. Finally we show the realization of a viscoelasto-plastic model which can be used, for example, to simulate the feel of a needle insertion procedure.

\subsubsection{Kelvin Model}

It is apparent from Fig. 8 and caption that the addition of physical dampers removes the delayrelated artifacts that occur on contact and release with a Kelvin object. Force spikes are clearly present in the traces when the manipulandum crosses $x=0$, and spikes results in the object feeling "sticky". This effect was previously noted by several researchers (Rosenberg and Adelstein, 1993). Following the initial contact with the Kelvin element, the manipulandum is released to show the damped recovery. With both physical and virtual damping, the recovery states look similar, and resemble the exponential function that represents the tissue recovery. The position traces are different because the experiment was performed manually.
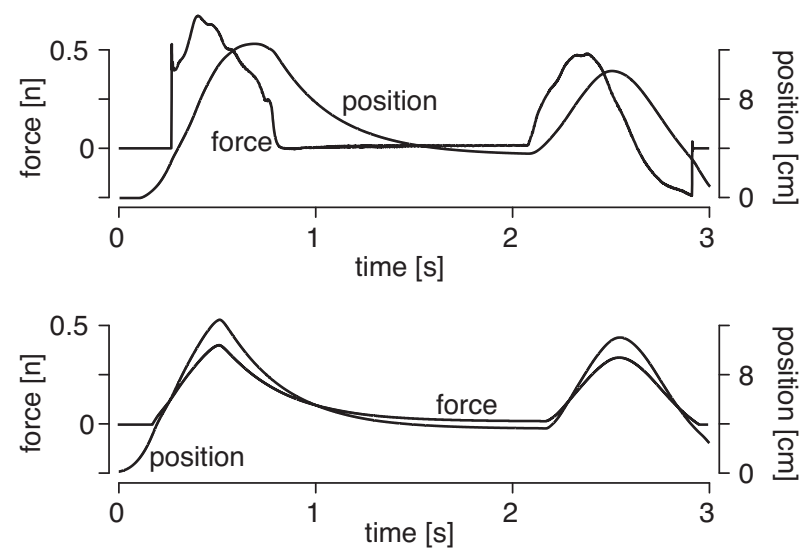

Figure 8: Kelvin viscolelasticity synthesized as per (9). The top panel shows the simulation realized computationally. The bottom panel shows the commanded torque to the motor. The viscous component is produced physically by the non-contact eddycurrent viscous dampers.

\subsubsection{Maxwell Model}

Fig. 9 shows synthesis results from a $2^{\text {nd }}$ order generalized Maxwell viscoelastic model as in Fig. 2e. In this experiment, a solid metallic obstacle was placed on the path of the manipulandum, inside the viscoelastic object, so it could be stopped sharply to show the relaxation characteristic of the model. Both the physical and virtually damped methods show an almost identical relaxation period. They are both physically realized by the motor. Since velocity changes abruptly, a discontinuity must appear somewhere in the simulation. The difference between the physical and virtually damped methods is apparent at the time of contact and under active compression of the virtual object. In the case of the virtually damped object, the shape of the curve resembles that shown in Fig. 5 (upper left panel). In the case of the physically damped object, the shape of the curve resembles that shown in Fig. 5 (lower right panel), as only the motor contribution can be monitored in realtime using the hardware setup. From these plots it is apparent that a dual-channel haptic interface does not have as clear reduction of artifacts compared to a single motor. Nevertheless, the physical viscous damping ensures passivity, and allows for stiff interactions with rigid objects, such as a bone. While this cannot be shown by plots, these sharp transients are actually not felt because their occurrence scoincide with the periods where the dampers are activated. 

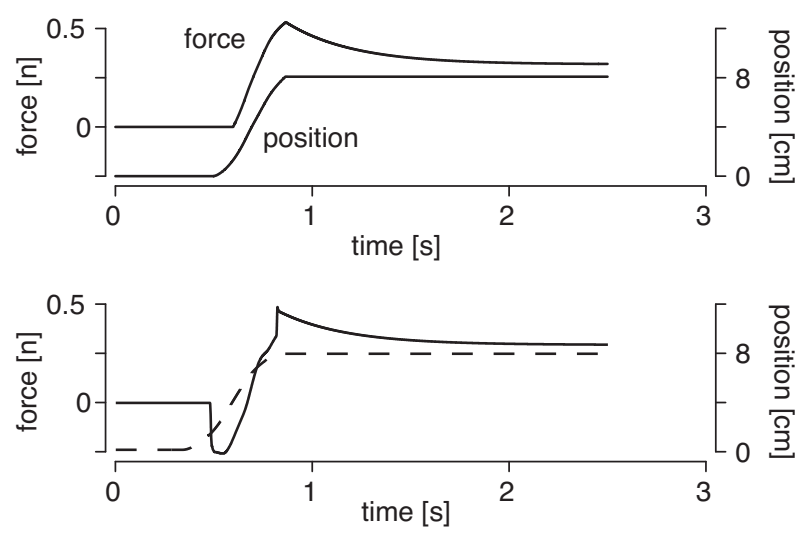

Figure 9: Maxwell viscolelasticity synthesized as per (19).The top panel shows the simulation realized computationally. The bottom panel shows the commanded torque to the motor. The rest is produced physically by the non-contact eddy-current viscous dampers.

\subsubsection{Visco-elasto-plastic model}

Percutaneous procedures are very common in modern medicine to deliver therapeutic material or sample tissues (Abolhassani et al., 2007). Procedures such as needle insertion (Alterovitz et al., 2003), catheter insertion (Gobbetti, 2000), or lumbar puncture (Gorman, 2000) are examples of medical simulations that could benefit from dual-channel haptics. Several authors have measured the forces of interaction of needles as they slide in biological tissues, typically in porcine liver (Barbé et al., 2007; Okamura et al., 2004; Hing et al., 2007). These studies show that a model which can describe the behavior of a needle moving inside a tissue must include a plastic component describing the sliding of the instrument in the tissues (irrecoverable displacement), an elastic component describing the deformation of surrounding tissues that can recover, and a viscous component that changes according to whether the instrument slides or is stuck. This general behavior can be modeled by the combination of elements shown in Fig. 10 which can represent visco-elastoplastic needle-tissue behavior.

The model shown in Fig. 10 corresponds to a viscoelastic, stip-slick friction model where the square box represents a plastic slip element. In the stuck state, it behaves like a Kelvin element with stiffness $K$ and damping $B_{1}$. During sliding, the nonlinear plastic slip element slides, making $\dot{x}=\dot{u}$. Thus, in

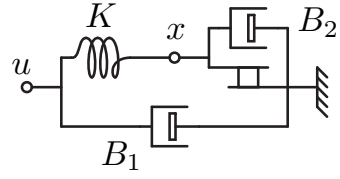

Figure 10: Visco-elasto-plastic model.

sliding, the output is the summation of a constant spring force, $K \delta_{\max }$, and the two dampers in parallel, $\dot{u}\left(B_{1}+B_{2}\right)$. The update law for this model is:

$$
f_{k+1}=\left\{\begin{array}{c}
K \delta_{\max }+\dot{u}_{k-1}\left(B_{1}+B_{2}\right) \\
x_{k+1}=u_{k}-\frac{\left|u_{k}-x_{k-1}\right|}{u_{k}-x_{k-1}} \delta_{\max } \\
\quad \text { if }\left|u_{k}-x_{k-1}\right|>\delta_{\max } \\
K\left(u_{k}-x_{k}\right)+B_{1} \dot{u}_{k-1}, \quad \text { otherwise }
\end{array}\right.
$$

where $\delta_{\max }$ is the spring deflection at the transition to sliding. Since our hardware does not have a force sensor, it has only a position sensor, and hence is of the impedance display type, $\delta_{\max }$ represents the presliding displacement in a manner similar to the friction synthesis algorithm described by Hayward and Armstrong (2000). Update law (21) can be separated into a elastic component to be realized by a motor and a viscous component to be realized by damper.

Fig. 11 shows the results of the synthesis of a viscoelasto-plastic model. In the computational version (upper panel), transient spikes occur with a delay after each velocity reversals. As expected from our findings where realizing a Kelvin element, these artifacts are eliminated when using the dual-channel haptic synthesis hardware. The haptic simulation using the viscous damper and motor combination improves the fidelity considerably and also guarantees passivity of a needle insertion simulation having visco-elasto-plastic components.

The elasto-visco-plastic element, in essence, models the local deformation of tissues interacting with an instrument. It could combined with other elements, specifically of the generalized Kelvin type (Section 5.1), to account for the global deformation deformation of an organ.

Due to the parallel nature of both the viscoelastic presliding and the viscoplastic sliding states of the model, a parallel attached motor/damper is ideally suited for display of such models. 

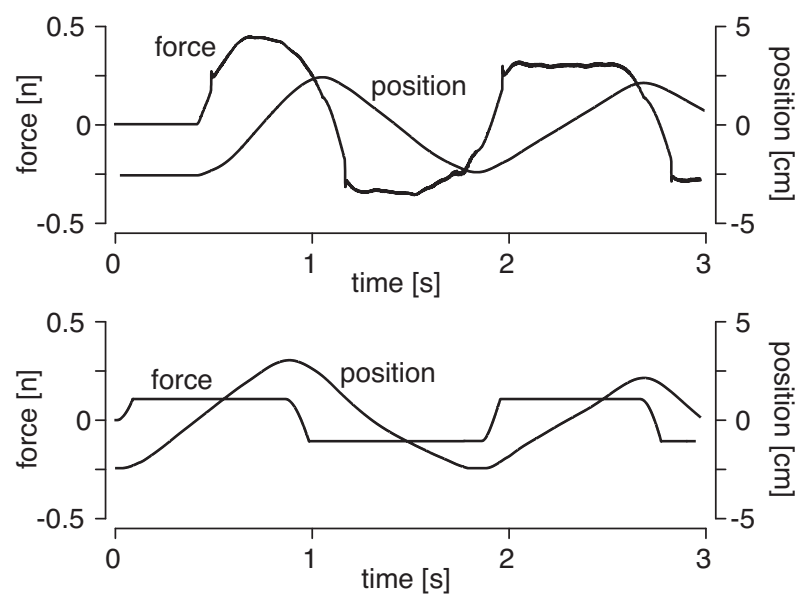

Figure 11: Visco-elasto-plastic model synthesized as per (21). The top pannel shows the torque command from the visco-elasto-plastic simulation realized computationally. The bottom pannel shows the dual-channel realization torque command.

\subsection{Energetic Behavior of Maxwell Ele- ments}

Simulation results from Section 5.4 showed that a delayed velocity estimation yielded extra energy at velocity reversals in a Maxwell element. In this section, we present experimental results that support this finding. Note that only Maxwell elements are analysed in detail, because the analysis of a Kelvin element has been studied in detail by previous researchers, for example by Colgate and Brown (1994).

We turn our attention to the energetic behavior of a Maxwell element. Of particular importance is the behavior at velocity reversals because this is where observation-based methods generate eroneous results. Fig. 12 shows experimental results of a Maxwell element at a velocity reversal, comparable to the simulation results described in Section 5.4. Shortly following the velocity reversal at approximately $3.12 \mathrm{~s}$, the Maxwell element changes from compression to elongation. It is clear from the figure that the virtually damped Maxwell element returns considerably more energy than the ideal case, while the dual-channel Maxwell element returns too little, which agrees with the previously discussed simulation results. For the experimental energy plots, a delay-free velocity signal was generated using first a non-causal 20 sample center-weighted averaging filter, followed by a $200 \mathrm{~Hz}$ Butterworth zero-delay digital filter implemented with the MATLAB $\AA$ Filter- ing Toolbox, zero phase function filtfilt(). This delay-free velocity signal was used to infer the contribution of the physical dampers necessary that is necessary to generate the dual-channel trace. It is important to note that the energy traces were computed offline, as it is not feasible to use non-causal, delay-free filters online.

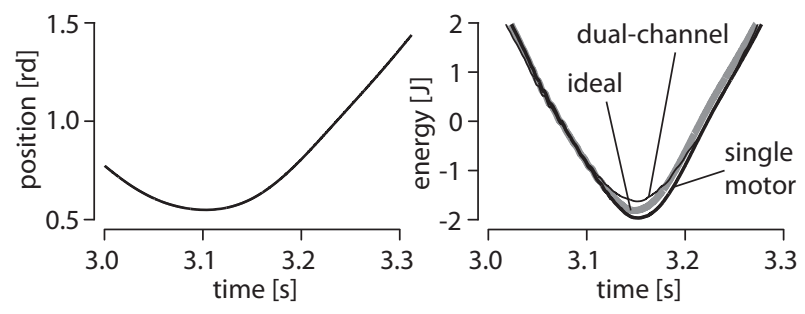

Figure 12: Energetic comparison at a velocity reversal of Maxwell element realizations. Thick grey line is ideal, thin black line is dual-channel, thick black line is single-motor actuation. As predicted, the single motor implementation displays active energetic behavior whereas the dual channel implementation is clearly passive.

From a separate test, Fig. 13 shows the results from a drag and release experiment done with a single Maxwell element. Upon release, it is clear that the dual-channel realization exhibits a more damped response, which yields fewer oscillations and a faster settling time compared to the more oscillatory behavior of the single motor realization. Because the experiments were performed manually, attention was paid to the initial conditions. At $4.2 \mathrm{~s}$ in the singlemotor, feedback-damped case, and $11.2 \mathrm{~s}$ in dualchannel, physically-damped plot, the release velocity was $1.8 \mathrm{rd} \cdot \mathrm{s}^{-1}$. Notice how the virtually damped element oscillates down to $-3.8 \mathrm{rd} \cdot \mathrm{s}^{-1}$, while the physically damped element reaches only $-2 \mathrm{rd} \cdot \mathrm{s}^{-1}$. The difference between these two results is entirely attributed to the delayed velocity estimation necessary for vibration-free display of a virtual damping coefficient.

\section{Conclusions And Future Work}

We introduced a method for the haptic synthesis of viscoelastic media using a dual-channel haptic interface. The prototype uses a DC motor as a programmable torquer in parallel with an ECB as a programmable viscous damper. This design is aimed 


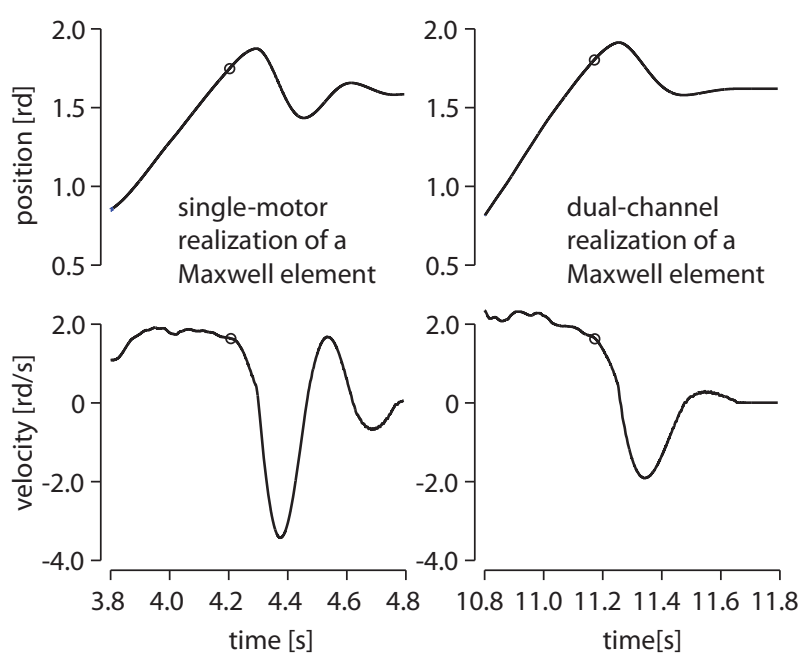

Figure 13: Drag and Release of a single Maxwell element. The left pannels show a virtually damped, single-motor realization of a Maxwell element. The right pannels show the physically damped, dualchannel realization of the same Maxwell element. The small circle indicates release of the manipulandum. In each case, the release velocity was nearly identical, approximately $1.8 \mathrm{rd} \cdot \mathrm{s}^{-1}$.

at avoiding or minimizing the dependence on a noisy and/or delayed velocity estimation signal. Due to the parallel nature of the device, viscoelastic functions that are connected in parallel, such as the Kelvin Model, or a visco-elasto-plastic model, can be simulated without velocity estimation in the feedback loop of the torquer. This approach minimizes artifacts that result from a delayed signal and actuator saturation.

For viscoelastic functions that are serial in nature, such as the Maxwell Model, the benefits of using the hybrid device are not as clear cut. A state must be simulated between each serially connected damperspring pair, and due to the purely dissipative nature of the ECB damper, the torquer must be used to compensate for the damper during transients. These transient torque corrections are velocity based, thus velocity estimation is required in the torquer feedback loop. The torquer corrections, however, are output when the physical viscous dampers are engaged, which minimizes the destabilizing effects that a delayed velocity signal can have on a haptic rendering and does not translate into tangible artifacts. Simulation and experimental results have shown that the dual-channel method improves the stability and passivity of a Maxwell element.

The examples we have given were produced with arbitrary viscoelastic parameters which may not match the medical reality. They were selected according to the capabilities of our available hardware and to exemplify the various properties of the dualchannel haptic synthesis approach. Medical procedures are numerous and varied, from orthopedic operations to retinal scrapping. It is clear that no single piece of haptic hardware will be capable to be adequately applied to the simulation of all possible cases. But starting from insertion procedures, to palpation, to more complex surgical gestures, one, two and higher numbers of actuated degrees of freedom in haptic devices could benefit from dual-channel actuation.

Our present efforts are directed at extending the hardware haptic realization theory and techniques to multi-dimensional cases, as per the recent work by Höver et al. (2009). This includes the specification of multidimensional visco-elasto-plastic functions, not at one single point of interaction, but anywhere on the surface or inside a virtual organ and the methods by which discontinuities can be handled without violations of hardware constraints such as actuator saturation and amplifier slew rates. An important aspect of a multidimensional synthesis of a force field is the generation of an arbitrary viscous field. While the theory behind the generation of such fields is not yet fully developed, preliminary results were shown in (Gosline et al., 2006), using motor corrections to eliminate the parasitic effects from coupled dampers. This approach requires further development and testing, and a method for online interpolation of dynamics response functions is required as well. There are also interesting new avenues to explore such as the design of compact and efficient ECBs. Preliminary engineering data indicates that such devices could be realized with form factors and masses comparable to the DC motors to which they could be optimally coupled.

\section{Acknowledgment}

This work was funded by a Collaborative Research and Development Grant "High Fidelity Surgical Simulation" from NSERC, the Natural Sciences and Engineering Council of Canada and by Immersion Corp., and by a Discovery Grant also from NSERC. 


\section{References}

Abolhassani, N., Patel, R., and Moallem, M. (2007). Needle Insertion Into Soft Tissue: A Survey. Medical Engineering and Physics 29:413-431.

Alterovitz, R., Pouliot, J., Taschereau, R., Hsu, I.C. J., and Goldberg, K. (2003). Simulating Needle Insertion And Radioactive Seed Implantation For Prostate Brachytherapy. In Proc. Medicine Meets Virtual Reality, Vol. 11, pp. 19-25.

Anwar, S. (2002). A Parametric Model Of An Eddy Current Electric Machine For Automotive Braking Applications. IEEE Transactions on Control Systems Technology 12(3):422-427.

Astley, O. R. and Hayward, V. (1998). Multirate Haptic Simulation Achieved By Coupling Finite Element Meshes Through Norton Equivalents. In Proc. IEEE International Conference on Robotics and Automation, pp. 989-994.

Basdogan, C., Ho, C. H., and Srinivasan, M. A. (2001). Virtual Environments For Medical Training: Graphical And Haptic Simulation Of Laparoscopic Common Bile Duct Exploration. IEEE/ASME Transactions on Mechatronics 6(3):269-285.

Basdogan, C., Sedef, M., Harders, M., and Wesarg, S. (2007). Virtual Reality Supported Simulators For Training In Minimally Invasive Surgery. IEEE Computer Graphics and Applications, 27(2):54-66.

Barbé, L., Bayle, B., de Mathelin, M., Gangi, A. (2007). Needle Insertions Modeling: Identifiability and Limitations. Biomedical Signal Processing and Control, 2(3):191-198.

Bélanger, P. R., Dobrovolny, P., Helmy, A., and Zhang, X. (1998). Estimation Of Angular Velocity And Acceleration From Shaft-Encoder Measurements. The International Journal of Robotics Research, 17(11):12251230.

Blackburn, J. F., Reethof, G., and Shearer, J. L. (1960). Fluid Power Control. MIT Press.

Brouwer, I., Mora, V., and Laroche, D. (2007). A Viscoelastic Soft Tissue Model For Haptic Surgical Simulation. In WHC '0\%: Proc. Second Joint EuroHaptics Conference and Symposium on Haptic Interfaces for Virtual Environment and Teleoperator Systems, pp. 593-594.

Cavusoglu, M. C., Feygin, D., and Tendick, F. (2002). A Critical Study Of The Mechanical And Electrical Properties Of The Phantom Haptic Interface And Improvements For High Performance Control.
Presence: Teleoperators and Virtual Environments 11(6):555-568.

Christensen, R. M. (1971). Theory of Viscoelasticity: An Introduction. Academic Press.

Colgate, J. E. and Brown, J. M. (1994). Factors Affecting The Z-Width Of A Haptic Display. In Proc. IEEE International Conference on Robotics and Automation, pp. 3205-3210.

Colgate, J. E. and Schenkel, G. (1994). Passivity Of A Class Of Sampled-Data Systems: Application To Haptic Interfaces. In Proc. American Control Conference, pp. 3236-3240.

Findley, W. N., Lai, J. S., and Onaran, K. (1976). Creep and Relaxation of Nonlinear Viscoelastic Materials with and Introduction to Linear Viscoelasticity. North-Holland Publishing Company.

Fung, Y. C. (1993). Biomechanics: Mechanical properties of living tissues. Springer-Verlag.

Gobbetti, E. (2000). Catheter Insertion Simulation With Co-Registered Direct Volume Rendering And Haptic Feedback. In Proc. Medicine Meets Virtual Reality, Vol. 8, pp. 96-98.

Goksel, O., Salcudean, S., and DiMaio, S. (2006). 3d Simulation Of Needle-Tissue Interaction With Application To Prostate Brachytherapy. Computer Aided Surgery 11(6):279-288.

Gorman, P. (2000). A Prototype Haptic Lumbar Puncture Simulator. In Proc. Medicine Meets Virtual Reality, Vol. 8, pp. 106-109.

Gosline, A. H., Campion, G., and Hayward, V. (2006). On The Use Of Eddy Current Brakes As Tunable, Fast Turn-On Viscous Dampers For Haptic Rendering. In Proc. Eurohaptics, pp. 229-234.

Gosline, A. H. and Hayward, V. (2008). Eddy Current Brakes For Haptic Interfaces: Design, Identification, And Control. IEEE/ASME Transactions on Mechatronics, in press.

Hannaford, B. and Ryu, J. H. (2002). Time-Domain Passivity Control Of Haptic Interfaces. IEEE Transactions on Robotics and Automation 18(1):1-10.

Harders, M. (2008). Haptics In Medical Applications. In Lin, M. and Otaduy, M., editors, Haptic Rendering: Foundations, Algorithms and Applications, A. K. Peters Ltd., pp. 589-612.

Hayward, V. (2007). Physically-Based Haptic Synthesis. In Lin, M. and Otaduy, M., editors, Haptic Rendering: Foundations, Algorithms and Applications, A. K. Peters Ltd., pp. 297-309. 
Hayward, V. and Armstrong, B. (2000). A New Computational Model Of Friction Applied To Haptic Rendering. In Corke, P. and Trevelyan, J., editors, Experimental Robotics VI, volume 250 of Lecture Notes in Control and Information Sciences, pp. 403-412.

Heald, M. A. (1988). Magnetic Braking: Improved Theory. American Journal of Physics 56(6):521-522.

Hing, J. T., Brooks, A. D., and Desai, J. P. (2007). A Biplanar Fluoroscopic Approach For The Measurement, Modeling, And Simulation Of Needle And Soft Tissue Interaction. Medical Image Analysis 11(1):6278.

Höver, R., Kósa, G., Sézkely, G, Harders, M. (2009). DataDriven Haptic Rendering from Viscous Fluids to Visco-Elastic Solids. IEEE Transactions on Haptics in press.

Janabi-Sharifi, F., Hayward, V., and Chen, C.-S. J. (2000). Discrete-Time Adaptive Windowing For Velocity Estimation. IEEE Transactions On Control Systems Technology 8(6):1003-1009.

Lederman, S. J., Klatzky, R. L., Hamilton, C. L., and Ramsay, G. I. (1999). Perceiving Roughness Via A Rigid Probe: Psychophysical Effects Of Exploration Speed And Mode Of Touch. Haptics-E: Electronic Journal of Haptics Research, 1.

Lee, K. and Park, K. (2002). Modeling Eddy Currents With Boundary Conditions By Sing Coulomb's Law And The Method Of Images. IEEE Transactions on Magnetics 38(2):1333-1340.

Okamura, A., Simone, C., and O'Leary, M. D. (2004). Force Modeling For Needle Insertion Into Soft Tissue. IEEE Transactions on Biomedical Engineering 51(10):1707-1716.

Rosenberg, L. B. and Adelstein, B. D. (1993). Perceptual Decomposition Of Virtual Haptic Surfaces. In Proc. Symposium on Research Frontiers in Virtual Reality, pp. 23-26.

Schoner, J. L. and Seidel, J. L. H. P. (2004). MeasurementBased Interactive Simulation Of Viscoelastic Solids. Computer Graphics Forum 23(3):547-556.

Sedef, M., Samur, E., and Basdogan, C. (2006). RealTime Finite-Element Simulation Of Linear Viscoelastic Tissue Behavior Based On Experimental Data. IEEE Computer Graphics and Applications 26(6):58-68.

Simeu, E. and Georges, D. (1996). Modeling And Control Of An Eddy Current Brake. Control Engineering Practise 4(1):19-26.
Su, Y., Zheng, C., Mueller, P., and Duan, B. (2006). A Simple Improved Velocity Estimation For LowSpeed Regions Based On Position Measurements Only. IEEE Transactions on Control Systems Technology 14(5):937-942.

Wallingford, E. E. and Wilson, J. D. (1977). HighResolution Shaft Speed Measurements Using A Microcomputer. IEEE Transactions On Instrumentation And Measurement IM-26(2):113-116.

Wang, Q. and Hayward, V. (2007). In Vivo Biomechanics Of The Fingerpad Skin Under Local Tangential Traction. Journal of Biomechanics, 40(4):851-860.

Weir, D. W., Colgate, J. E., and Peshkin, M. A. (2008). Measuring And Increasing Z-Width With Active Electrical Damping. In Proc. of the Symposium on Haptic Interfaces for Virtual Environments and Teleoperator Systems, pp. 169-175.

Wiederick, H. D., Gauthier, H., Campbell, D. A., and Rochon, P. (1987). Magnetic Braking: Simple Theory And Experiment. American Journal of Physics 55(6):500-503. 